INPLASY

PROTOCOL

To cite: Liu et al. Comparative effectiveness of adjuvant treatment for curative resected hepatocellular carcinoma: A network meta-analysis. Inplasy protocol 2020110039. doi: 10.37766/inplasy2020.11.0039

Received: 09 November 2020

Published: 10 November 2020

Corresponding author: Ying Liu

liuying2e@163.com

Author Affiliation:

Zhongshan Hospital; Fudan

University

Support: NSFC.

Review Stage at time of this submission: Preliminary searches.

Conflicts of interest: The authors declare no conflict of interest.

\section{Comparative effectiveness of adjuvant treatment for curative resected hepatocellular carcinoma: $\mathbf{A}$ network meta-analysis}

Liu, Y1; Wang, Y2; Lv, Q3; Li, X4; Huang, X5.

Review question / Objective: The aim is to compare the relative efficacy and the ranking probabilities of adjuvant therapies in curative resected patients with HCC. Condition being studied: Curative resected HCC.

Information sources: We will systematically searched (up to November, 2020) PubMed, Embase and Cochrane Library. We will also manually searched the relevant systematic reviews for potentially eligible articles.

INPLASY registration number: This protocol was registered with the International Platform of Registered Systematic Review and Meta-Analysis Protocols (INPLASY) on 10 November 2020 and was last updated on 10 November 2020 (registration number INPLASY2020110039).

\section{INTRODUCTION}

Review question / Objective: The aim is to compare the relative efficacy and the ranking probabilities of adjuvant therapies in curative resected patients with HCC.

Condition being studied: Curative resected HCC.

\section{METHODS}

Search strategy: We systematically searched (up to November, 2020) PubMed, Embase and Cochrane Library. We also manually searched the relevant systematic reviews for potentially eligible articles. The searches will be refined using the Boolean term "AND" between three parts: "liver 
cancer", "hepatocellular carcinoma", "HCC", "hepatic carcinoma", "hepatoma"; "adjuvant", "post-operative", "postoperative"; "randomized controlled trial".

Participant or population: Patients with HCC who had undergone a curative hepatectomy.

Intervention: Adjuvant therapy after hepatectomy.

Comparator: Hepatectomy alone.

Study designs to be included: Randomized controlled trial.

Eligibility criteria: (1) randomized controlled trials; (2) patients with HCC who had undergone a curative hepatectomy; (3) reported at least one clinical outcome of interest including recurrence or overall survival (OS).

Information sources: We will systematically searched (up to November, 2020) PubMed, Embase and Cochrane Library. We will also manually searched the relevant systematic reviews for potentially eligible articles.

Main outcome(s): Recurrence.

Additional outcome(s): Overall survival.

Quality assessment / Risk of bias analysis: Seven items specifically developed from the Cochrane risk of bias tools were used by two reviewers (Ying Liu; Yuzhu Wang) to assess the quality of the eligible studies. Any discrepancies in data extraction and quality assessment were resolved by discussion in the whole study groups.

Strategy of data synthesis: First, traditional pairwise meta-analysis that directly compared interventions with observation were performed. The statistical heterogeneity in each pairwise comparison was evaluated using 12 statistic with $p$ values. A fixed-effect models were employed, if $\mathrm{I} 2<50 \%$, otherwise a randomeffect models was used. Secondly, we used Stata (version 15.0) to generate the network meta diagram, in which nodes and edges revealed the head-to-head comparisons among interventions. Thirdly, the NMA was conducted in the Bayesian framework using a Markov Chain Monte Carlo technique in OpenBUGS (version 3.2.3).

Subgroup analysis: None.

Sensibility analysis: Sensibility analysis will be conducted to check the robustness and reliability of pooled outcome results.

Country(ies) involved: China.

Keywords: Hepatocellular carcinoma; Adjuvant therapy; Network meta-analysis.

Contributions of each author:

Author 1 - Ying Liu.

Author 2 - Yuzhu Wang.

Author 3 - Qianzhou Lv.

Author 4 - Xiaoyu Li.

Author 5 - Xiaowu Huang. 